\title{
CyLineUp: A Cytoscape app for visualizing data in network
}

\section{small multiples [version 1; peer review: 2 approved]}

\section{Maria Cecília D. Costa ${ }^{*}$, Thijs Slijkhuis ${ }^{2 *}$, Wilco Ligterink ${ }^{1}$, Henk W.M. Hilhorst ${ }^{1}$, Dick de Ridder², Harm Nijveen (1D1,2}

${ }^{1}$ Wageningen Seed Lab, Laboratory of Plant Physiology, Wageningen University, Wageningen, The Netherlands

${ }^{2}$ Bioinformatics Group, Wageningen University, Wageningen, The Netherlands

* Equal contributors

\section{V1 First published: $11 \mathrm{Apr}$ 2016, 5:635 \\ https://doi.org/10.12688/f1000research.8402.1 \\ Latest published: 11 Apr 2016, 5:635 \\ https://doi.org/10.12688/f1000research.8402.1}

\section{Abstract}

CyLineUp is a Cytoscape 3 app for the projection of high-throughput measurement data from multiple experiments/samples on a network or pathway map using "small multiples". This visualization method allows for easy comparison of different experiments in the context of the network or pathway. The user can import various kinds of measurement data and select any appropriate Cytoscape network or WikiPathways pathway map. CyLineUp creates small multiples by replicating the loaded network as many times as there are experiments/samples (e.g. time points, stress conditions, tissues, etc.). The measurement data for each experiment are then mapped onto the nodes (genes, proteins etc.) of the corresponding network using a color gradient. Each step of creating the visualization can be customized to the user's needs. The results can be exported as a high quality vector image.

\section{Keywords}

Data display , Expression profiling , Graphical user interfaces , Metabolite profiling, Small multiples

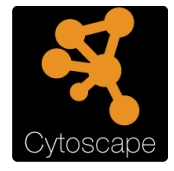

This article is included in the Cytoscape

gateway.

\section{Open Peer Review}

Approval Status

1 2

version 1

11 Apr 2016

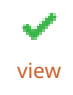

\section{$\checkmark$
view}

1. Alexander Pico id Gladstone Institutes, San Francisco, USA

2. Oren Tzfadia, Ghent University, Ghent, Belgium

Any reports and responses or comments on the article can be found at the end of the article. 
Corresponding author: Harm Nijveen (harm.nijveen@wur.nl)

Competing interests: No competing interests were disclosed.

Grant information: This work has been supported by the 'Conselho Nacional de Desenvolvimento Científico e Tecnológico' (CNPq, Brazil) (MCDC).

Copyright: $@ 2016$ Costa MCD et al. This is an open access article distributed under the terms of the Creative Commons Attribution License, which permits unrestricted use, distribution, and reproduction in any medium, provided the original work is properly cited.

How to cite this article: Costa MCD, Slijkhuis T, Ligterink W et al. CyLineUp: A Cytoscape app for visualizing data in network small multiples [version 1; peer review: 2 approved] F1000Research 2016, 5:635 https://doi.org/10.12688/f1000research.8402.1

First published: 11 Apr 2016, 5:635 https://doi.org/10.12688/f1000research.8402.1 


\section{Introduction}

Developments in high-throughput -omics measurement techniques have allowed researchers to routinely obtain presence/absence, levels or interactions of molecules at a genome-wide scale, often addressing important questions that could not be answered before. Consequently, the demand for more and better tools for data analysis is growing, especially visualization tools to project data of multiple samples (e.g. different time points or tissues) on network or pathway maps. Visualization plays an important role in data analysis as a way to obtain insights, select details and present findings ${ }^{1}$.

Several software tools enable visualization of expression data on pathways ${ }^{2-4}$ and the functionality to plot charts on nodes has become a standard feature of Cytoscape $3^{5}$. While these visualizations often work well for analyzing measurement patterns of individual genes in a network, they do not easily show changes at a more global level in the network. Often, these global effects are the most interesting to the biologist.

Here, we present a different approach that uses "small multiples". This approach is a visualization technique that shows multiple copies of a graph with the same combination of variables, but with different changes in each variable ${ }^{6}$. Small multiples facilitate comparison, presentation, storytelling and search for patterns, trends and outliers ${ }^{1}$.

There is currently no visualization tool enabling projection of data from different experiments/samples on small multiples. Therefore, we developed the Cytoscape app CyLineUp, that takes advantage of the tools for importing and displaying maps integrated into
Cytoscape to create small multiples. We applied CyLineUp to visualize changes in the glycolysis/tricarboxylic acid (TCA) cycle induced by the phytohormone abscisic acid (ABA) in germinated Arabidopsis thaliana seeds.

\section{Methods and implementation \\ Implementation}

CyLineUp was written in Java 7 as an OSGi (Open Services Gateway Initiative) bundle. It adds a "CyLineUp" tab to the Cytoscape "Control Panel" offering functionality to import the data, configure the views and visual styles, and export an SVG rendering of the visualization. The data import functionality is implemented as a Cytoscape Task, using Tunable annotations for user input dialogs. The small multiples visualization is implemented as a set of views on the same underlying network. Each view has its own visual style for coloring the nodes according to the input data. The FreeHEP Graphics 2D Library is used for exporting an SVG image with the small multiples.

\section{Operation}

Visualizing -omics data with CyLineUp consists of four main steps. (I) Creating the map or network that will be the basis for data visualization. A large collection of pathways from the WikiPathways platform (http://wikipathways.org) can be easily imported using the WikiPathways Cytoscape app ${ }^{7}$. Optionally, pathways created and imported directly from Pathvisio ${ }^{8}$ and networks created in Cytoscape can be used. The users should take care that the names of the identifiers are the same in the data file and in the pathway/ network. (II) Importing data and linking it to the map using the graphical user interface provided by CyLineUp (Figure 1).

A

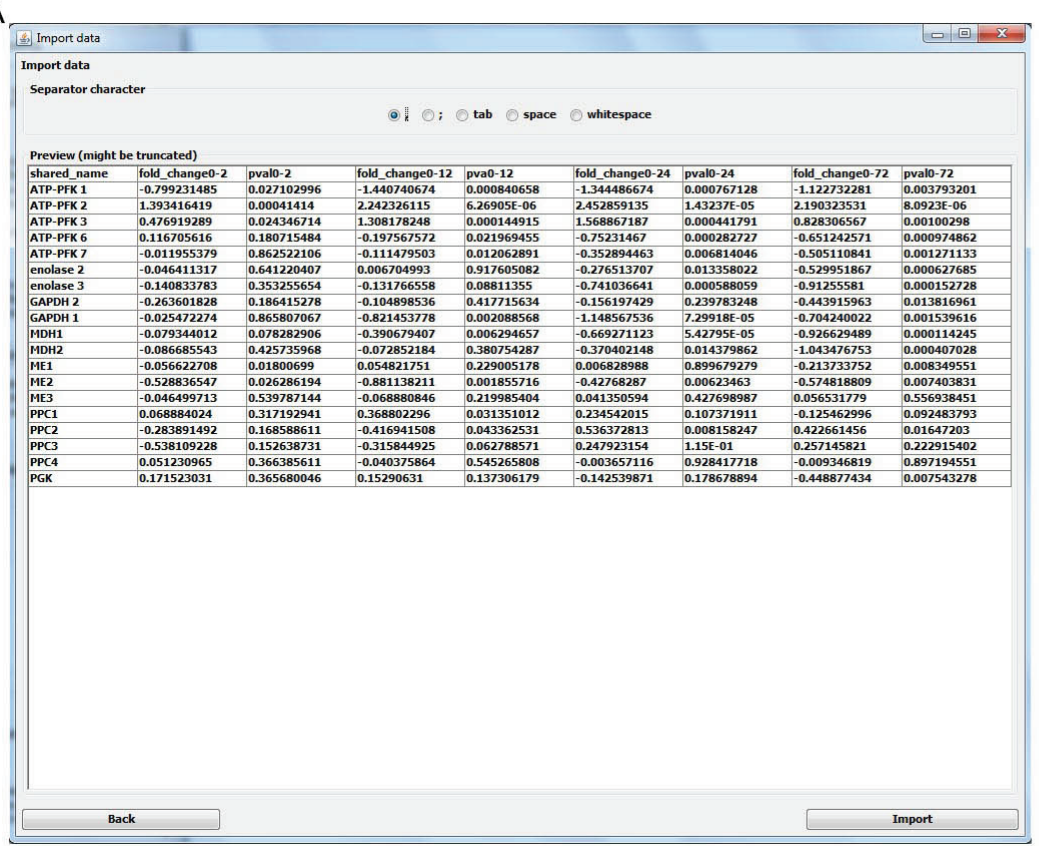

B

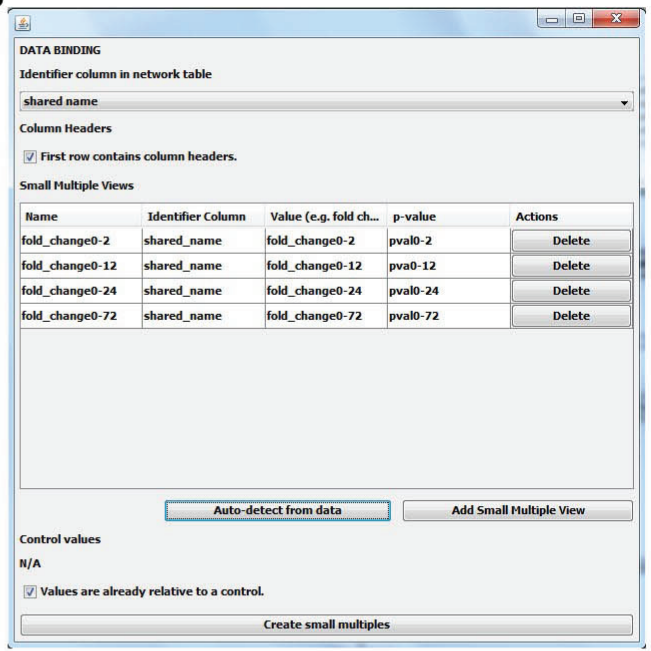

Figure 1. Importing data. (A) After selecting the text file with the input data, the user should select the field/column separator character (comma, semicolon, tab or whitespace). (B) On the data binding panel, the user should select the column that contains the identifier ("shared name" in our example) and configure the small multiples either by using the auto-detect functionality or manually (add small multiple views). 
CyLineUp accepts text files with comma, semicolon, tab or arbitrary whitespaces separated values. CyLineUp provides autoimport functionality, but manual selection of data is also possible. The auto-import functionality is based on the order of the columns in the data file. Therefore, it is advisable to have the identifiers in the first column and the values (e.g. fold change) and the p-values for each treatment/sample in subsequent columns. (III) Customizing the visualization to the user's needs by changing the Visual styles in the CyLineUp user interface (Figure 2). Nodes that have no data associated can be shown (default), greyed out or hidden. In case the nodes are hidden, the edges associated to them will also be hidden. The default colors are green (HEX \#00FF00) for up-regulated genes and red (HEX \#FF0000) for down-regulated genes. The user can chose different colors by pressing the Pick color buttons. After each change, the user can update the view of the small multiples by pressing the button Update network views. (IV) Exporting the visualization to an SVG image file. A real-time image preview is available, but can be disabled in case it takes too much computer resources.

\section{Use case}

We used available gene expression data from a time series of microarrays ${ }^{9}$ to validate the use of CyLineUp. The data were generated to explore the temporal changes induced by ABA in germinated A. thaliana seeds ${ }^{9}$. Seeds at the stage of radicle protrusion $(0 \mathrm{~h})$ and after four periods $(2,12,24$ and $72 \mathrm{~h})$ of incubation in ABA were used. Details about data acquisition and processing can be found in Costa et al. ${ }^{9}$. Here, we used CyLineUp to explore the main changes in the glycolysis/TCA cycle during ABA incubation (Figure 3).

The map of the cycle was created using Pathvisio 3.2.0 and imported into Cytoscape 3.2.1 (Supplementary file S1.gpml). Then, the expression data and Bonferroni-corrected p-values (commaseparated values (CSV) file: Supplementary file S2.csv) were imported using the CyLineUp user interface. The visual style was set to "grey out" nodes that have no data associated, use a "p-value cut-off" of 0.1 and "use fill color to visualize p-value".

The glycolysis/TCA cycle provides most of the energy for processes in the seed. A general activation of the cycle could be observed as one of the immediate cellular responses induced by $\mathrm{ABA}^{10}$.

\section{Conclusion}

CyLineUp allows easy visual analysis of data from multiple samples on a biological network to study changes at the (sub-)network level. It is a useful and timely addition to the Cytoscape network analysis platform.

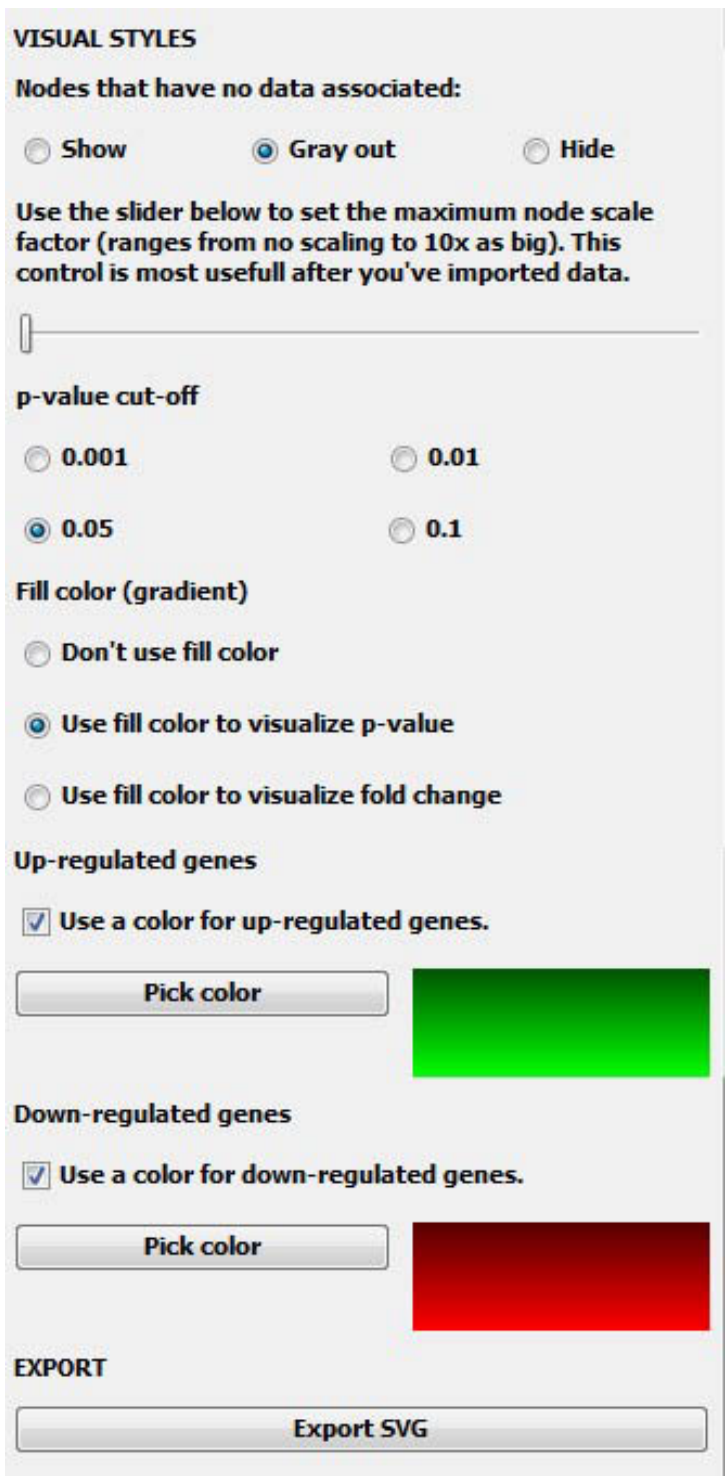

Figure 2. Configuring the visual styles. The user can customize several visualization settings by changing the Visual styles. Nodes that have no data associated can be shown (default), greyed out or hidden. For hidden nodes, the associated edges will also be hidden. The node color can be set to reflect the p-value cut-off $(0.001,0.01$, 0.05 and 0.1 ) or fold change. The default colors are green (HEX \#00FF00) for "up-regulated genes" and red (HEX \#FF0000) for "down-regulated genes". The user can choose different colors by pressing the Pick color buttons. After customization, the user can export the visualization to an SVG image file. 

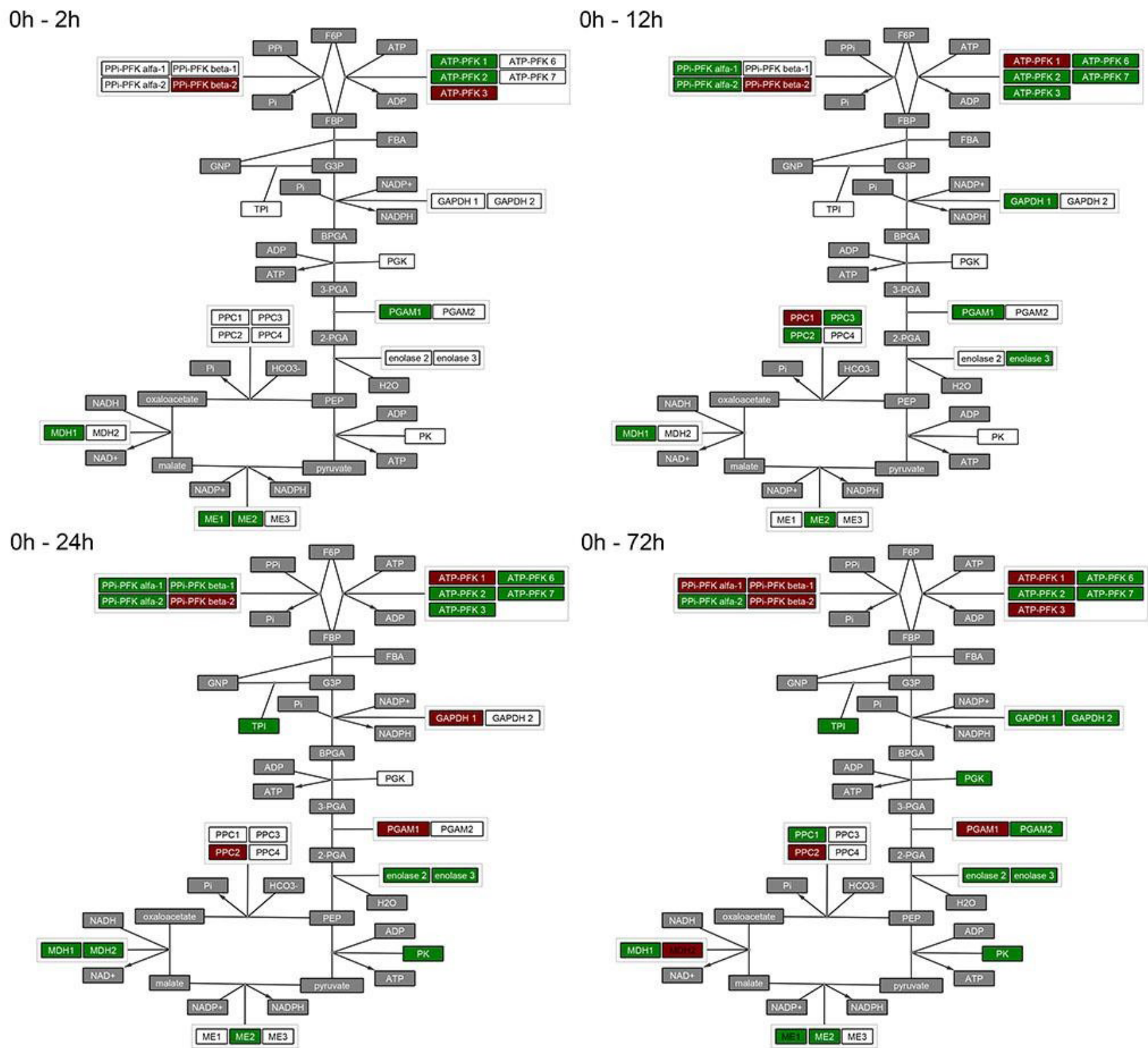

Figure 3. Small multiples showing temporal changes in the expression of genes acting in the glycolysis/TCA cycle induced in

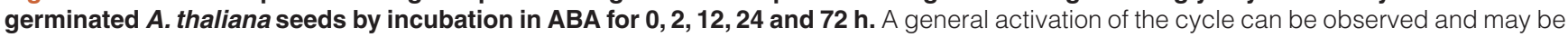
a mechanism by which the plant cell prepares for the energy demands of recovery from drought. Red nodes indicate genes with significantly declining transcript abundance. Green nodes indicate genes with significantly accumulating transcripts. White nodes indicate genes without significant changes in transcript abundance. Grey nodes indicate components that are not genes or do not have data associated. 


\section{Software availability}

Software available from: http://apps.cytoscape.org/apps/cylineup

Latest source code: https://github.com/Slijkhuis/CyLineUp

Archived source code as at time of publication: http://dx.doi. org/10.5281/zenodo. $48135^{11}$

License: Lesser GNU Public License 2.1

Author contributions

MCDC wrote the manuscript and provided experimental data to validate the use of the app. TS developed initial version of the app. WL, HWMH and DDR provided input on the manuscript. HN supervised app development, improved the app and provided input on the manuscript.

\section{Competing interests}

No competing interests were disclosed.

Grant information

This work has been supported by the 'Conselho Nacional de Desenvolvimento Científico e Tecnológico’ (CNPq, Brazil) (MCDC).

\section{Supplementary material}

Supplementary file S1.gpml Map of the glycolysis/TCA cycle.

Supplementary file S2.csv Expression values and Bonferroni-corrected p-values.

1. van den Elzen S, van Wijk JJ: Small multiples, large singles: a new approach for visual data exploration. Comput Graph Forum. 2013; 32(3pt2): 191-200. Publisher Full Text

2. Westenberg MA, Roerdink JB, Kuipers OP, et al:: SpotXplore: a Cytoscape plugin for visual exploration of hotspot expression in gene regulatory networks. Bioinformatics. 2010; 26(22): 2922-3.

PubMed Abstract | Publisher Full Text | Free Full Text

3. Yamada T, Letunic I, Okuda S, et al.: iPath2.0: interactive pathway explorer. Nucleic Acids Res. 2011; 39(Web Server issue): W412-5. PubMed Abstract | Publisher Full Text | Free Full Text

4. Thimm O, Bläsing O, Gibon Y, et al.: MAPMAN: a user-driven tool to display genomics data sets onto diagrams of metabolic pathways and other biological processes. Plant J. 2004; 37(6): 914-39. [cited 2011 Aug 4]. PubMed Abstract | Publisher Full Text

5. Shannon P, Markiel A, Ozier O, et al: Cytoscape: a software environment for integrated models of biomolecular interaction networks. Genome Res. 2003 13(11): 2498-504.

PubMed Abstract | Publisher Full Text | Free Full Text
6. Tufte ER: The visual display of quantitative information. In: Tufte ER, editor The visual display of quantitative information. 2nd ed. Cheshre, Connecticut, Connecticut: Graphics Press, 2001; 197. Reference Source

7. Kutmon M, Riutta A, Nunes N, et al.: WikiPathways: capturing the full diversity of pathway knowledge. Nucleic Acids Res. 2016; 44(D1): D488-94 PubMed Abstract | Publisher Full Text | Free Full Text

8. Kutmon M, van lersel MP, Bohler A, et al: PathVisio 3: an extendable pathway analysis toolbox. PLOS Comput Biol. 2015; 11(2): e1004085. PubMed Abstract | Publisher Full Text | Free Full Text

9. Costa MC, Nijveen $\mathrm{H}$, Ligterink W, et al:: Time-series analysis of the transcriptome of the re-establishment of desiccation tolerance by ABA in germinated Arabidopsis thaliana seeds. Genom Data. 2015; 5: 154-6. Elsevier B.V. PubMed Abstract | Publisher Full Text | Free Full Text

10. Toldi $O$, Tuba $Z$, Scott $P$ : Vegetative desiccation tolerance: Is it a goldmine for bioengineering crops? Plant Sci. 2009; 176(2): 187-99. [cited 2011 Aug 7]. Publisher Full Text

11. Costa MC, Slijkhuis T, Nijveen H: CyLineUp. Zenodo. 2016. Data Source 


\section{Open Peer Review}

\section{Current Peer Review Status:}

\section{Version 1}

Reviewer Report 14 June 2016

https://doi.org/10.5256/f1000research.9038.r14169

(C) 2016 Tzfadia 0. This is an open access peer review report distributed under the terms of the Creative Commons Attribution License, which permits unrestricted use, distribution, and reproduction in any medium, provided the original work is properly cited.

\section{Oren Tzfadia}

Department of Plant Biotechnology and Bioinformatics, Ghent University, Ghent, Belgium

CyLineUp is a new Cytoscape app which allow users to visualize 'changing' networks by enabling users to match dynamic features to networks.

In my humble opinion, I find the title a bit confusing - and would modify it to something like:

"CyLineUp: A Cytoscape app for vizualization of data-matched style in multiple networks".

The abstract does a fair job in describing the main features of the app presented in the article. The article include suffice explanations, and usage examples, of the app with real biological data. The app itself is very easy to install and use - and thus is very user friendly, which is always an advantage as Cytoscape is heavily used by no-computer-expert biologists. From my own experience, by following the screenshots and explanations provided in the article, it is easy to plot your own data with CyLineUp.

I see the CyLineUp app as a potentially great app for analyzing multiple 'omics' data sets, as often we wish to plot small multiples based on different omics data sets.

Competing Interests: No competing interests were disclosed.

I confirm that I have read this submission and believe that I have an appropriate level of expertise to confirm that it is of an acceptable scientific standard.

Reviewer Report 19 April 2016

https://doi.org/10.5256/f1000research.9038.r13301

(C) 2016 Pico A. This is an open access peer review report distributed under the terms of the Creative Commons Attribution License, which permits unrestricted use, distribution, and reproduction in any medium, provided the original work is properly cited. 


\section{Alexander Pico \\ Gladstone Institutes, San Francisco, CA, USA}

It is a common use case for Cytoscape, to have data for multiple conditions or time points. There are a few other apps to address this in various ways, including cyAnimator, enhancedGraphics and DynNetwork, which utilize either animation or custom chart graphics for nodes. The "small multiples" approach has always been an option for users who were sufficiently skilled with using Cytoscape, which allows one to make multiple networks and apply a unique, data-mapped style to each. This is a tedious and error-prone task, however. So, the CyLineUp app is a welcome tool to make this task easy, consistent and reliable.

The app is easy to install and use, and the paper is clear and well-written. It is nice to see a fundamental visualization technique like "small multiples" applied here. However, it is disappointing to see the red-green color gradient offered by default, which should be shunned by data visualization tool developers. Perhaps something to consider changing in the next release of the app? Since the app is open source, maybe I'll contribute this code patch myself as a pull request!

The CyLineUp app offers its own table importer, visual style editor and image exporter customized for visualizing data for multiple conditions or time points. Looking forward, it would be interesting to see what would be involved in adapting the core of Cytoscape to be able to annotate columns of table data already imported and then support the small multiple view generator, leveraging the native importer, style editor and image exporters. I could picture this functionality becoming a part of the Cytoscape distribution, in the same way the enhancedGraphics app was eventually merged with the core as an out-of-the-box feature. This would make the useful facility of small multiple views available to all users, without having to learn these new, single-purpose interfaces.

Competing Interests: Non-financial competing interests: I reviewed an earlier version of the paper as guest editor for this collection of Cytoscape app articles. I am a core developer of Cytoscape. I am also a developer of WikiPathways, which is mentioned and cited in the article. I believe these interests did not bias my review beyond informing my perspective on the usefulness of the tool, but I'll leave that judgement to you, the reader of these open peer reviews.

I confirm that I have read this submission and believe that I have an appropriate level of expertise to confirm that it is of an acceptable scientific standard. 
The benefits of publishing with F1000Research:

- Your article is published within days, with no editorial bias

- You can publish traditional articles, null/negative results, case reports, data notes and more

- The peer review process is transparent and collaborative

- Your article is indexed in PubMed after passing peer review

- Dedicated customer support at every stage

For pre-submission enquiries, contact research@f1000.com 